\title{
Microfluidics for reconfigurable electromagnetic metamaterials
}

T. Serkan Kasirga, Y. Nuri Ertas, and Mehmet Bayindir

Citation: Appl. Phys. Lett. 95, 214102 (2009);

View online: https://doi.org/10.1063/1.3268448

View Table of Contents: http://aip.scitation.org/toc/apl/95/21

Published by the American Institute of Physics

\section{Articles you may be interested in}

Structural tunability in metamaterials

Applied Physics Letters 95, 084105 (2009); 10.1063/1.3211920

Electrically tunable negative permeability metamaterials based on nematic liquid crystals Applied Physics Letters 90, 011112 (2007); 10.1063/1.2430485

Magnetic control of negative permeability metamaterials based on liquid crystals Applied Physics Letters 92, 193104 (2008); 10.1063/1.2926678

Tunable optical negative-index metamaterials employing anisotropic liquid crystals Applied Physics Letters 91, 143122 (2007); 10.1063/1.2795345

A reconfigurable liquid metal antenna driven by electrochemically controlled capillarity Journal of Applied Physics 117, 194901 (2015); 10.1063/1.4919605

Liquid crystal based nonlinear fishnet metamaterials

Applied Physics Letters 100, 121113 (2012); 10.1063/1.3695165

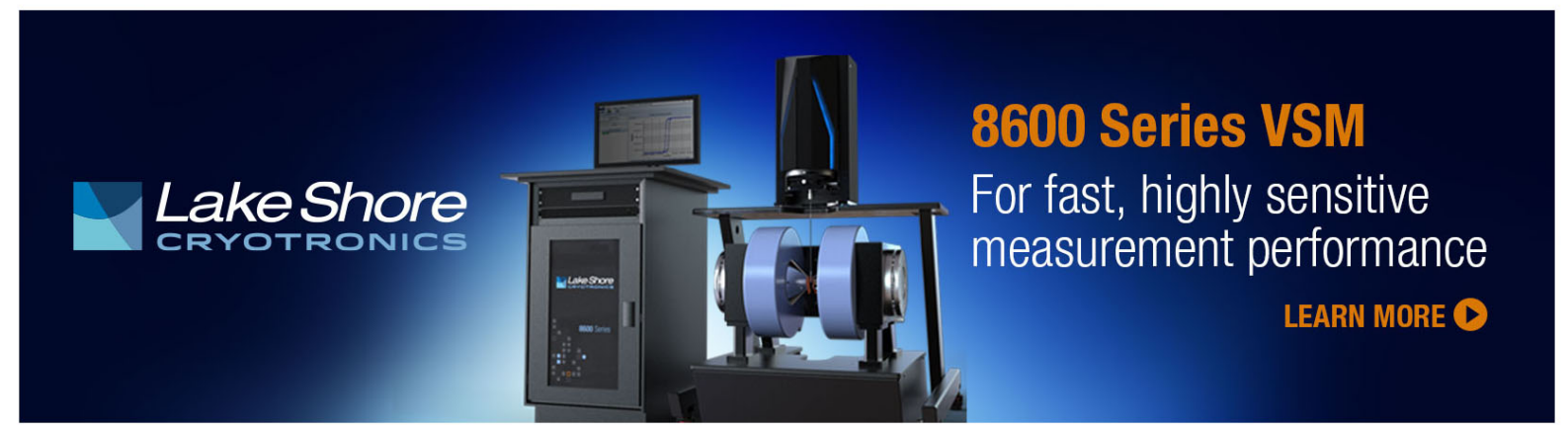




\title{
Microfluidics for reconfigurable electromagnetic metamaterials
}

\author{
T. Serkan Kasirga, ${ }^{1,2}$ Y. Nuri Ertas, ${ }^{1}$ and Mehmet Bayindir ${ }^{1,2, a)}$ \\ ${ }^{1}$ UNAM-Institute of Materials Science and Nanotechnology, Bilkent University, 06800 Ankara, Turkey \\ ${ }^{2}$ Department of Physics, Bilkent University, 06800 Ankara, Turkey
}

(Received 7 September 2009; accepted 3 November 2009; published online 25 November 2009)

\begin{abstract}
We propose microfluidics as a useful platform for reconfigurable electromagnetic metamaterials. Microfluidic split-ring resonators (MF-SRRs) are fabricated inside a flexible elastomeric material by employing rapid prototyping. The transmission measurements performed for mercury-injected MF-SRR exhibits sharp magnetic resonances at microwave wavelengths. We further calculate transmission properties of the MF-SRR array and the effect of electrical conductivity of the liquid inside the channel on the magnetic resonance. The measured results agree well with numerical calculations. Our proposal may open up directions toward switchable metamaterials and reconfigurable devices such as filters, switches, and resonators. (C) 2009 American Institute of Physics. [doi:10.1063/1.3268448]
\end{abstract}

Left-handed metamaterials (LHMs) having periodically arranged split-ring resonators (SRRs) (Refs. 1-3) and thin wires $^{4}$ offer vast variety of applications due to their peculiar electromagnetic (EM) properties. ${ }^{5-8}$ Recently, tunable LHM components have attracted great interest since some of the potential applications of LHM require dynamical control of the EM properties. ${ }^{9-18}$ Because, the frequency region with negative permeability $(-\mu)$ is narrow compared to negative permittivity $(-\varepsilon$,$) region, left-handed passband frequency re-$ gion mostly depend on the magnetic plasma frequency $\left(\omega_{m}\right)$ of the SRR lattice. ${ }^{1,2}$ Thus, being able to tune $\omega_{m}$ will lead to dynamically tunable metamaterials. The magnetic resonance frequency of SRRs can be tuned by various mechanisms such as capacitor loaded SRRs, ${ }^{9,10,12,13}$ photoconductive semiconductors, ${ }^{11}$ nematic liquid crystals, ${ }^{14,18}$ and ferroelectric loaded split rings. ${ }^{16}$ The electrical resonance of SRR structures can also be tuned in order to modulate optical properties of devices. ${ }^{19}$

In this letter, we propose and demonstrate a new platform for metamaterial components based on microfluidics. Microfluidic split-ring resonator (MF-SRR) is fabricated in an elastomeric material [poly(dimethylsiloxane) (PDMS)] by employing rapid prototyping. Transmission measurements are performed for a mercury-injected single MF-SRR, and a magnetic resonance is observed at microwave frequencies. We further investigate the transmission properties of an MFSRR array and the effect of the electrical conductivity of injected liquid inside MF channels on the magnetic resonance by using finite difference time domain (FDTD) simulations.

We use rapid prototyping ${ }^{20}$ which is a common method to fabricate devices in microfluidics, ${ }^{21}$ to fabricate MF-SRR channels in PDMS polymer as shown in Figs. 1(a)-1(h). Single-SRR geometry ${ }^{6}$ is preferred due to the ease of metallic liquid injection. In this geometry, $\omega_{m}$ can be tuned by changing the structural parameters $c$ and $h$ [Fig. 1(i)]. Two monopole antennas are used to measure the transmission properties of MF-SRR in free space [Fig. 1(j)]. First, we measured the EM response of a single MF-SRR with and without mercury injection through the microfluidic channels.

\footnotetext{
${ }^{a)}$ Electronic mail: bayindir@nano.org.tr.
}
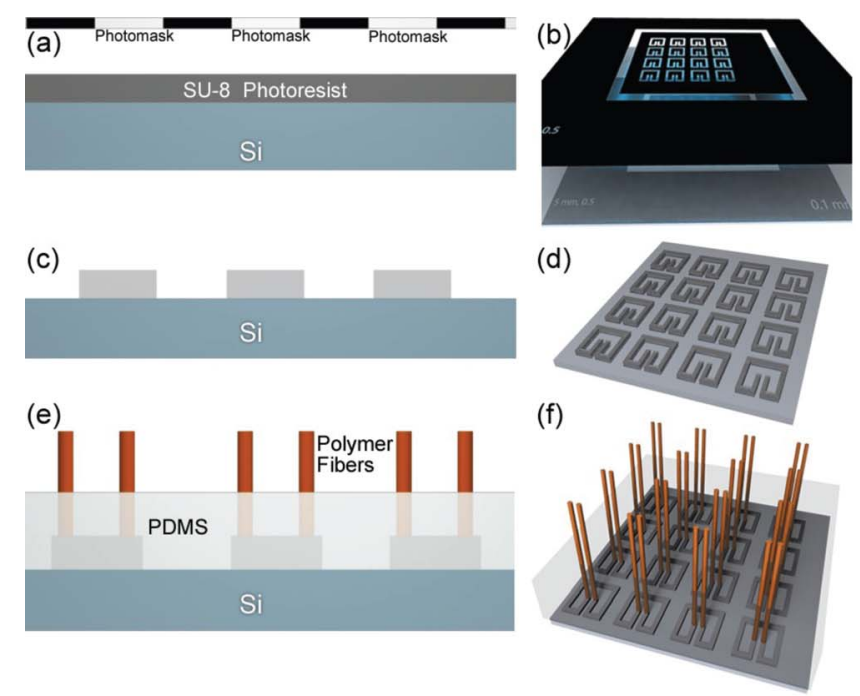

(g)
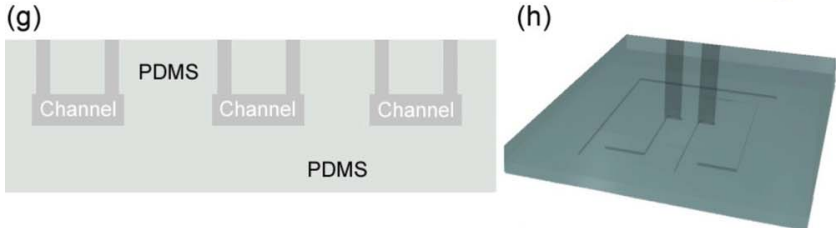

(i)
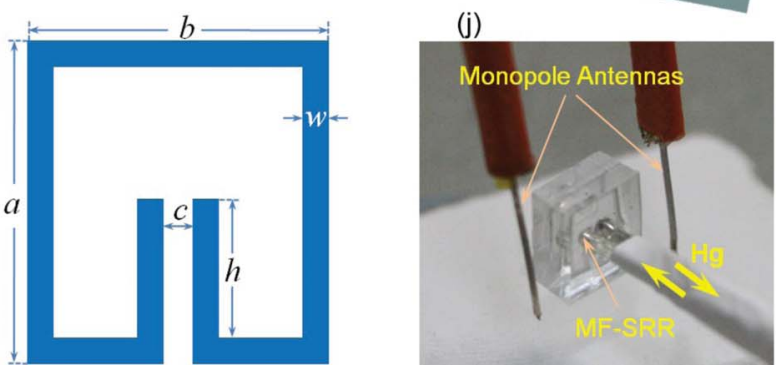

FIG. 1. (Color online) Fabrication steps of MF-SRRs. [(a) and (b)] MF-SRR patterns on SU-8 50 photoresist, by using photolithography. [(c) and (d)] Defining positive relief of MF-SRR array on Si wafer. [(e) and (f)] Placing polymer fibers to define in and out channels. Casting PDMS onto Si wafer, then curing the polymer at $65^{\circ} \mathrm{C}$ and peeling cured PDMS from the wafer. Polymer fibers are obtained from a macroscopic preform by thermal drawing in a custom-build fiber tower. (g) Sealing of SRRs with bottom PDMS slab after exposing both surfaces to oxygen plasma. (h) A single MF-SRR cut from the MF-SRR array is ready for metal injection. (i) Schematic drawing of a MF-SRR with parameters $a=5.5 \mathrm{~mm}, b=5.0 \mathrm{~mm}, h=2.4 \mathrm{~mm}, c$ $=0.7 \mathrm{~mm}$, and $w=0.5 \mathrm{~mm}$. (j) Microwave transmission measurement setup with two monopole antennas. 


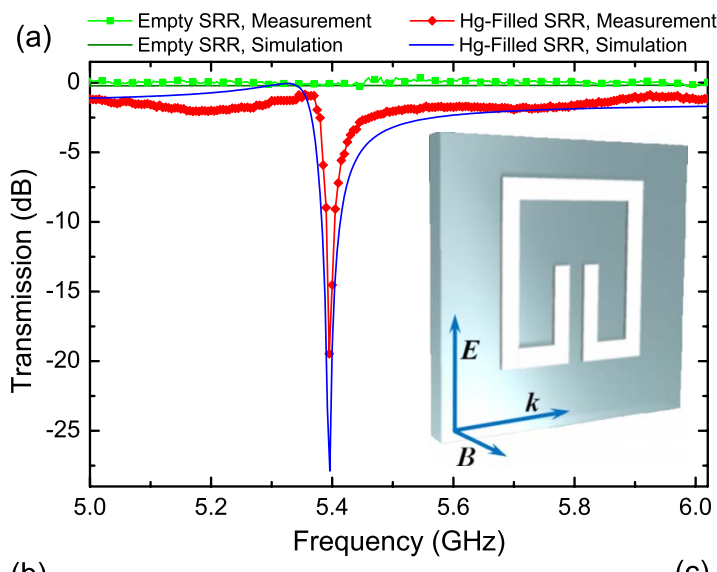

(b)

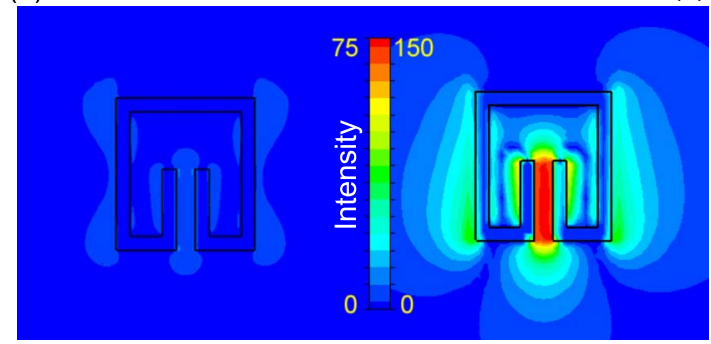

FIG. 2. (Color online) (a) Measured and calculated transmission spectrum of a single MF-SRR before and after metal injection. A magnetic resonance exists at $\omega=5.41 \mathrm{GHz}$. The inset shows orientation of MF-SRR structure. Calculated electric field intensity at (b) off-resonance frequency $\omega$ $=7.0 \mathrm{GHz}$ and (c) on-resonance frequency $\omega=5.41 \mathrm{GHz}$.

Figure 2(a) displays the measured and calculated responses of empty and mercury-filled MF-SRR. Mercury-filled MFSRR exhibits a sharp magnetic resonance at $5.41 \mathrm{GHz}$, while empty MF-SRR the spectra shows no resonances, as expected. The FDTD simulation result agrees well with the measurements. In simulations, the conductivity of mercury is taken $\sigma_{\mathrm{Hg}}=10^{6} \mathrm{~S} / \mathrm{m}$. Since the inlet and outlet channels are perpendicular to MF-SRR plane, they do not affect the resonance frequency $\omega_{m}$.

Appearance of the magnetic resonance in transmission spectrum after metal injection into MF-SRR channel indicates the negative permeability which allows to tune EM properties dynamically. The switching time between on $(-\mu)$ and off $(+\mu)$ modes of MF-SRR is order of seconds and limited by the pumping speed of mercury to the channel. It is not observed any significant change in the magnetic resonance frequency and deformation of channels after repeating the experiments several times. It is important to note that switching of magnetic resonance frequency is not possible in previously proposed tunable SRR structures; in these studies frequency can only be tuned within a narrow frequency range.

Figures 2(b) and 2(c) show calculated electric field intensity distribution in a single MF-SRR at off- and onresonance frequencies, respectively. While the field intensity is very weak at the split region of SRR at off-resonance frequency $(\omega=7.0 \mathrm{GHz})$, the field intensity increased and mostly concentrated at the split region at on-resonance frequency $(\omega=5.41 \mathrm{GHz})$.

The effective permeability of an MF-SRR array can be estimated from following expression: ${ }^{1}$

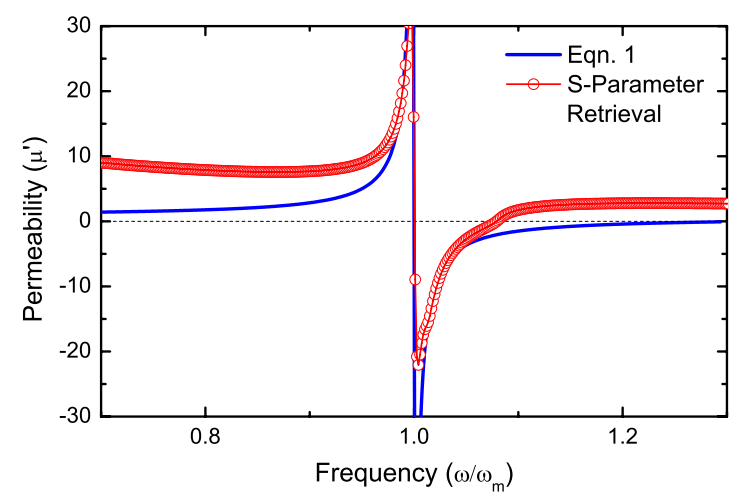

FIG. 3. (Color online) Calculated real part of effective permeability of mercury-filled MF-SRR medium as a function of normalized frequency by using Eq. (1) and S-parameter retrieval procedure.

$$
\mu_{\mathrm{eff}}(\omega)=\mu^{\prime}+i \mu^{\prime \prime}=1-\frac{F \omega^{2}}{\omega^{2}-\omega_{m}^{2}+i \Gamma \omega},
$$

where $\omega$ is the frequency of incident radiation and $F=0.43$ is the fractional area of the unit cell occupied by the interior of a MF-SRR and $\Gamma=0.12 \mathrm{GHz}$ is the dissipation factor. Figure 3 displays the calculated real part of $\mu_{\text {eff }}$ using Eq. (1) and standard S-parameter retrieval method. ${ }^{22}$ It can be seen that the negative magnetic response region extends up to $6 \mathrm{GHz}$.

We also calculated the transmission properties of mercury-filled and empty MF-SRR array (Fig. 4). While empty MF-SRR array does not show any resonances, mercury-filled MF-SRR array exhibits a stop band region centered at $5.2 \mathrm{GHz}$. The SRR array is embedded inside PDMS polymer. The simulated result indicates the possibility of obtaining $-\mu$ and $+\mu$ media, and switching between each others in flexible substrates.

The MF-SRR can also be filled with by any other conducting liquids such as colloidal silver solution containing nanoparticles. Since conductivity of metallic liquid is critical for obtaining magnetic resonances in MF-SRR structures, we investigated the effect of conductivity of the liquid used in MF-SRR on the magnetic resonance $\omega_{m}$. As shown in Fig. 5, the magnetic resonance diminishes significantly as the conductivity of the liquid decreases. The quality factor $Q$ $=\omega_{m} / \Delta \omega$ of magnetic resonance decreases rapidly with decreasing conductivity (Fig. 5 inset).

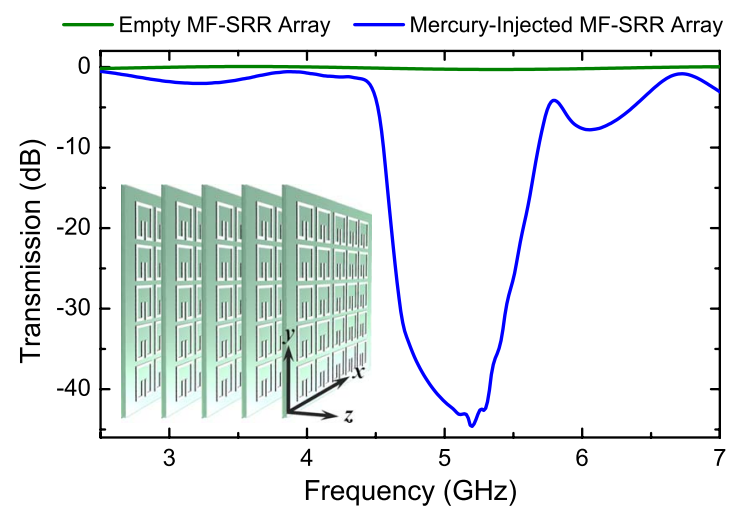

FIG. 4. (Color online) Calculated transmission spectrum of mercury-filled MF-SRR medium and empty MF-SRR with periods $a_{x}=6.2 \mathrm{~mm}, a_{y}$ $=6.7 \mathrm{~mm}$, and $a_{z}=5.0 \mathrm{~mm}$. A negative permeability region is centered around $5.2 \mathrm{GHz}$. The inset shows schematic drawing of mercury-filled MFSRR medium. E-field polarization is along $+y$ direction. 


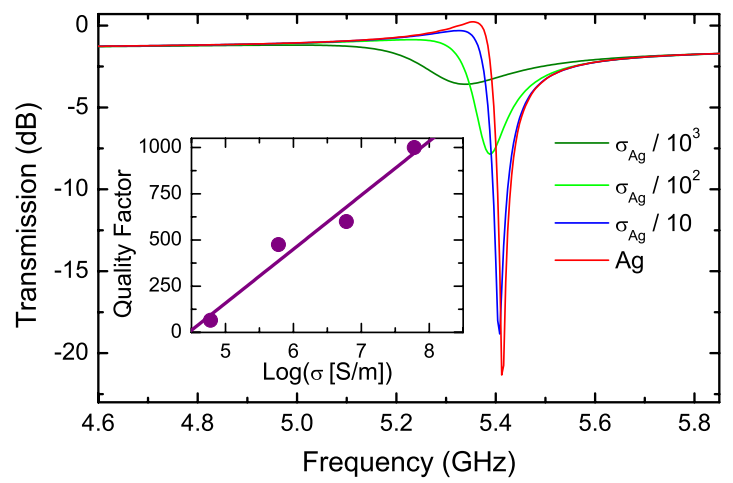

FIG. 5. (Color online) Calculated transmission spectrum of a MF-SRR as a function of electrical conductivity, $\sigma$, of liquid injected into the channels. Inset shows the effect of conductivity of the fluid inside the MF-SRR on the quality factor of the MF-SRR resonance.

In the present work, we reported the possibility of achieving reconfigurable metamaterial components in flexible polymer substrates. The negative permeability region can be tuned by adding another microfluidic channel in the split region of MF-SRR. By injecting liquids with different permittivity and permeabilities inside this channel one can modify the capacitance of the MF-SRR, consequently the magnetic resonance frequency can be tuned. ${ }^{23}$ Combining negative permitivity $(-\varepsilon)$ with negative permeability $(-\mu)$ it should be possible to obtain reconfigurable MF-LHM. Thus, with the ability of microfluidic metamaterials it is possible to realize switchable EM medium, i.e., switching left-handed medium to right-hanged medium or vice versa. In addition, MF-SRR may be useful platform for construction of reconfigurable devices such as filters, switches, and resonators.

This work is supported by TUBITAK under the Grant No. 106G090. MB acknowledges support from the Turkish Academy of Sciences Distinguished Young Scientist Award (TUBA GEBIP). This work was performed at UNAMInstitute of Materials Science and Nanotechnology supported by the State Planning Organization of Turkey through the
National Nanotechnology Research Center Project. We thank H. Caglayan and S. Cakmakyapan from Ozbay Research Group for their help in microwave measurements.

${ }^{1}$ J. B. Pendry, A. J. Holden, D. J. Robbins, and W. J. Stewart, IEEE Trans. Microwave Theory Tech. 47, 2075 (1999).

${ }^{2}$ D. R. Smith, W. J. Padilla, D. C. Vier, S. C. Nemat-Nasser, and S. Schultz, Phys. Rev. Lett. 84, 4184 (2000)

${ }^{3}$ M. Bayindir, K. Aydin, E. Ozbay, P. Markos, and C. M. Soukoulis, Appl. Phys. Lett. 81, 120 (2002).

${ }^{4}$ J. B. Pendry, A. J. Holden, W. J. Stewart, and I. Youngs, Phys. Rev. Lett. 76, 4773 (1996).

${ }^{5}$ D. R. Smith, J. B. Pendry, and M. C. K. Wiltshire, Science 305, 788 (2004).

${ }^{6}$ D. Schurig, J. J. Mock, B. J. Justice, S. A. Cummer, J. B. Pendry, A. F. Starr, and D. R. Smith, Science 314, 977 (2006).

${ }^{7}$ V. M. Shalaev, Nat. Photonics 1, 41 (2006).

${ }^{8}$ R. Marques, F. Martin, and M. Sorolla, Metamaterials with Negative Parameters: Theory, Design and Microwave Applications (Wiley, New Jersey, 2008).

${ }^{9}$ I. Gil, J. Bonache, J. Garcia-Garcia, and F. Martin, IEEE Trans. Microwave Theory Tech. 54, 2665 (2006).

${ }^{10}$ I. V. Shadrivov, S. K. Morisson, and Y. S. Kivshar, Opt. Express 14, 9344 (2006).

${ }^{11}$ A. Degiron, J. J. Mock, and D. R. Smith, Opt. Express 15, 1115 (2007).

${ }^{12}$ K. Aydin and E. Ozbay, J. Appl. Phys. 101, 024911 (2007).

${ }^{13}$ D. Wang, L. Ran, H. Chen, M. Mu, J. A. Kong, and B. Wu, Appl. Phys. Lett. 91, 164101 (2007).

${ }^{14}$ Q. Zhao, L. Kang, B. Du, B. Li, J. Zhou, H. Tang, X. Liang, and B. Zhang, Appl. Phys. Lett. 90, 011112 (2007).

${ }^{15}$ Q. Zhao, B. Du, L. Kang, H. Zhao, B. Li, X. Zhang, J. Zhou, L. Li, and Y. Meng, Appl. Phys. Lett. 92, 051106 (2008).

${ }^{16}$ T. H. Hand and S. A. Cummer, J. Appl. Phys. 103, 066105 (2008).

${ }^{17}$ L. Kang, Q. Zhao, H. Zhao, and J. Zhou, Opt. Express 16, 8825 (2008).

${ }^{18}$ F. Zhang, Q. Zhao, L. Kang, D. P. Gaillot, X. Zhao, J. Zhou, and S. Lippens, Appl. Phys. Lett. 92, 193104 (2008).

${ }^{19}$ H.-T. Chen, W. J. Padilla, J. M. O. Zide, A. C. Gossard, A. J. Taylor, and R. D. Averitt, Nature (London) 444, 597 (2006).

${ }^{20}$ D. C. Duffy, J. C. McDonald, O. J. A. Schueller, and G. M. Whitesides, Anal. Chem. 70, 4974 (1998).

${ }^{21}$ N.-T. Nguyen and S. T. Wereley, Fundamentals and Applications of Microfluidics, 2nd ed. (Artech House, Boston, 2006).

${ }^{22}$ D. R. Smith, D. C. Vier, Th. Koschny, and C. M. Soukoulis, Phys. Rev. E 71, 036617 (2005).

${ }^{23}$ T. S. Kasirga and M. Bayindir (unpublished). 